\title{
Molecular mechanisms of chemotropism and cell fusion in unicellular fungi
}

\author{
Sophie G. Martin
}

\begin{abstract}
In all eukaryotic phyla, cell fusion is important for many aspects of life, from sexual reproduction to tissue formation. Fungal cells fuse during mating to form the zygote, and during vegetative growth to connect mycelia. Prior to fusion, cells first detect gradients of pheromonal chemoattractants that are released by their partner and polarize growth in their direction. Upon pairing, cells digest their cell wall at the site of contact and merge their plasma membrane. In this Review, I discuss recent work on the chemotropic response of the yeast models Saccharomyces cerevisiae and Schizosaccharomyces pombe, which has led to a novel model of gradient sensing: the cell builds a motile cortical polarized patch, which acts as site of communication where pheromones are released and sensed. Initial patch dynamics serve to correct its position and align it with the gradient from the partner cell. Furthermore, I highlight the transition from cell wall expansion during growth to cell wall digestion, which is imposed by physical and signaling changes owing to hyperpolarization that is induced by cell proximity. To conclude, I discuss mechanisms of membrane fusion, whose characterization remains a major challenge for the future.
\end{abstract}

KEY WORDS: Cell fusion, Cell polarity, Chemotropism, Pheromone gradient, Cdc42, MAPK, S. cerevisiae, S. pombe

\section{Introduction}

All cells - whether they live on their own or are part of a multicellular organism - interact with their environment. They are able to perceive neighbors and respond to external signals that guide their behavior. More often than not, the perceived signals are directional and lead to a polar cellular response that is critical for cell physiology. Examples of such chemotropic behaviors include those of leukocytes migrating towards and responding to antigens, amoeba responding to a cue for aggregation, neurons extending growth cones to form neural networks, pollen tubes growing in the stigma towards the egg cell or fungal hyphae creating connected mycelia (Artemenko et al., 2014; Palanivelu and Preuss, 2000; Roca et al., 2005). The encounter of gametes during sexual reproduction is a particularly important form of chemotropic behavior, which culminates in their fusion to form the zygote. This is likely to be a very ancient process, as sex is thought to have evolved in the last common eukaryotic ancestor and is present in all extant eukaryotic phyla (Goodenough and Heitman, 2014).

In this Review, I present our current knowledge on the chemotropic response of unicellular fungi (specifically the ascomycetes Saccharomyces cerevisiae and Schizosaccharomyces pombe) during sexual reproduction and its climax in cell fusion. Department of Fundamental Microbiology, University of Lausanne, 1015 Lausanne,
Switzerland.

*Author for correspondence (sophie.martin@unil.ch)

(D) S.G.M., 0000-0002-5317-2557
I will further draw comparison to somatic fusion in filamentous fungi. Recent work has fundamentally changed our view of how these cells, which detect chemoattractants through canonical eukaryotic signaling pathways, orient up a gradient: instead of simultaneously comparing chemoattractant concentration around themselves, they organize a subcellular detection zone that sequentially probes the concentration in their periphery.

\section{A primer on fungal pheromone signaling and cell fusion}

Yeast cells signal to each other and induce sexual differentiation through the secretion of pheromones. Pheromone signaling is very similar in $S$. cerevisiae and $S$. pombe, with some notable differences. In $S$. cerevisiae, a- and $\alpha$-cells (M- and P-cells in $S$. pombe) secrete short peptide pheromones, of which the a-factor (M-factor) is lipid modified. Pheromones are recognized by cognate G-protein-coupled receptors (GPCR) on the partner cell. Although pheromones and their receptors are cell type specific, pheromone engagement activates a mitogen-activated protein kinase (MAPK) signaling pathway that is common to both cell types. Receptor activation promotes the exchange of GDP for GTP on the coupled $\mathrm{G} \alpha$ subunit and, in $S$. cerevisiae, dissociation from the G $\beta \gamma$ complex, which is now free to activate MAPK signaling by recruiting the MAPK scaffold Ste5 to the membrane [see Alvaro and Thorner (2016) for a complete recent review on GPCR signaling in $S$. cerevisiae]. In $S$. pombe, receptor activation also elicits activation of a MAPK cascade, which is under dual control of G $\alpha$ and a Ras GTPase. In this case, G $\alpha$ plays a positive role in signal transduction, and, in fact, the nature of the G $\beta \gamma$ complex, if any, remains elusive (Merlini et al., 2013) (Fig. 1).

The fusion of fungi - non-motile cells encased in rigid cell walls can be divided into three stages. First, the cells signal their position by acting as source of pheromone and polarize growth towards each other to achieve cell contact. The ability of cells to orient growth in response to an external cue, also called chemotropism, requires the coupling of two basic functionalities: detection of the pheromones by receptors and polarized growth. In yeast, polarized growth depends on the local activation of the GTPase $\mathrm{Cdc} 42$, which drives polarized secretion. The molecular links between the two functionalities is best understood in $S$. cerevisiae, where G $\beta \gamma$ released from G $\alpha$ inhibition upon receptor activation forms a complex through Far1 with a Cdc42 guanine nucleotide exchange factor (GEF) (Cdc24 in $S$. cerevisiae, hereafter referred to as the Cdc42 GEF). In S. pombe, the molecular connection is not deciphered but likely involves the Ras family GTPase Ras1, which, in addition to its function as MAPK activator, promotes Cdc42 activation (Fig. 1). Second, once cells have paired, which also involves cell surface adhesin molecules, they need to digest their cell wall at the zone of contact, leading to plasma membrane apposition. Because the yeast cell wall offers protection not only against external insults but also against lysis due to high internal turgor pressure, the time and location of wall digestion has to be controlled. Third, the plasma membranes merge together to unite 


\section{S. cerevisiae}

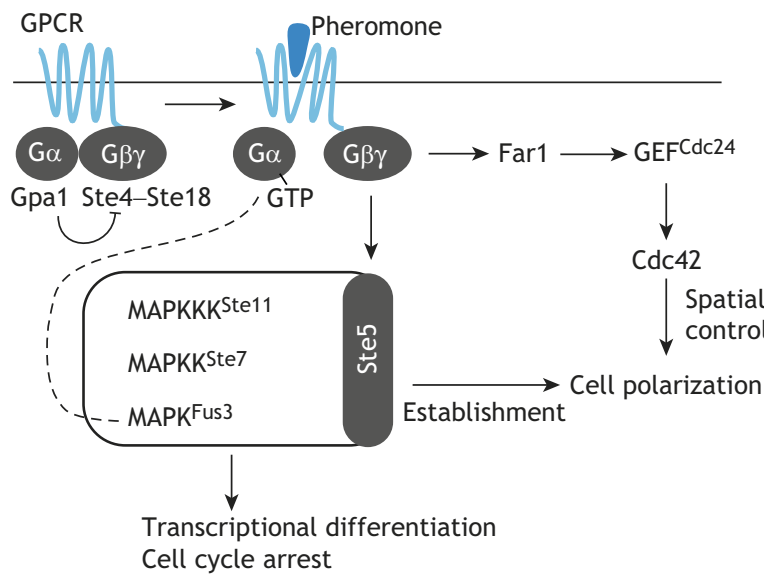

\section{S. pombe}

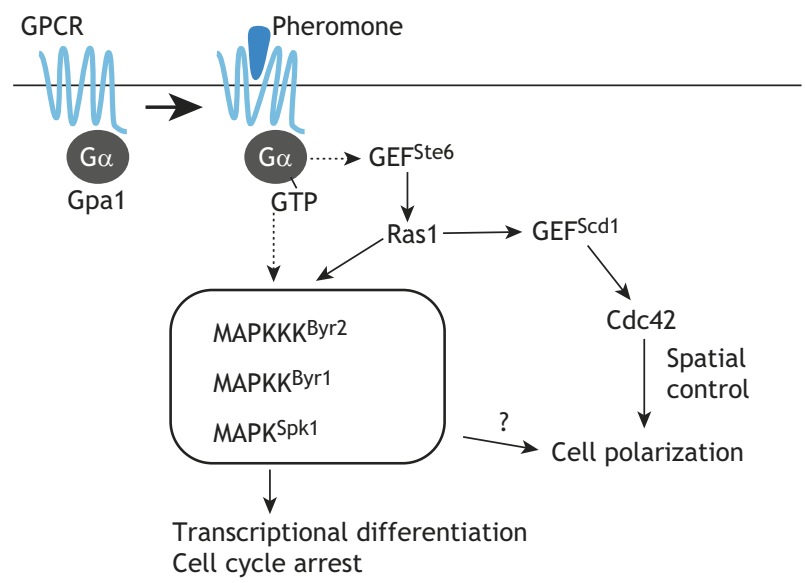

Fig. 1. Schematic depiction of pheromone-sensing pathways in yeasts. Pheromone binding to the receptor leads to G $\alpha$ GTP binding. In S. cerevisiae, this causes dissociation from G $\beta \gamma$, which is now free to activate the MAPK cascade to induce cell differentiation, cell cycle arrest and initiation of cell polarization. $\mathrm{G} \beta$ also forms a complex with the Cdc42 GEF with the help of Far1 to provide a positional bias to Cdc42 activation. In addition, G $\alpha$ directly binds the MAPK Fus3 (dashed line). In S. pombe, the activated $\mathrm{G} \alpha$ signals positively to activate the MAPK cascade. The molecular links are unknown (dotted lines) but involve Ras-dependent and Ras-independent routes. Ras both acts as MAPK activator and forms a complex with the Cdc42 GEF to couple cell polarization to pheromone perception.

the two cells as one. This entire process takes $1-2 \mathrm{~h}$ in $S$. cerevisiae, for which presence of a mating partner induces differentiation immediately after the cell completes its last division (Henderson et al., 2018preprint). In $S$. pombe, the process is slower: cells initiate differentiation only upon starvation, divide once or twice, and then pair and fuse over the next 10-12 h (Vjestica et al., 2016).

\section{Challenges for spatial decoding}

The classical view of chemotropism in yeast (Arkowitz, 2009) was inspired by the spatial decoding mechanism of chemotactic amoeba (see Box 1). Indeed, the identification of specific $S$. cerevisiae alleles that prevent the interaction of the Cdc42 GEF with $\mathrm{G} \beta \gamma$ revealed that this physical link underlies chemotropism (Butty et al., 1998; Nern and Arkowitz, 1998, 1999). This led to the interpretation that the site of pheromone sensing marked by free G $\beta \gamma$ acts as a landmark for recruitment of the Cdc42 GEF. Spatial decoding requires comparison of receptor occupancy between the front and the back of the cell. However, because yeast cells are small (of the order of 4-5 $\mu \mathrm{m}$ ), yet able to detect shallow gradients, the difference in occupancy may be minute. Indeed, use of quantitatively defined gradients in microfluidic devices suggests that a $0.5 \%$ to $5 \%$ difference in pheromone concentration between the cell front and back is sufficient for accurate gradient detection, which requires a very strong amplification system to yield robust polarization (Moore et al., 2008; Muller et al., 2016). Note that these two studies reached distinct conclusions regarding the range of pheromone concentrations that leads to robust directional growth. Spatial sensing also necessitates pheromone receptors to be present around the entire cell periphery. Accordingly, in S. cerevisiae, Ste2 (the $\alpha$-factor receptor expressed in a-cells) is constitutively expressed and present over the entire cell surface in mitotically proliferating cells. However, pheromones trigger the rapid endocytosis of Ste2 with a halftime of $\sim 6$ min (Emmerstorfer-Augustin et al., 2018), which then becomes enriched at the shmoo (the mating projection) cortex $\sim 30 \mathrm{~min}$ later. This observation poses a significant challenge to the idea of spatial decoding, as even at this late stage, the cell is able to start a new site of growth if the gradient direction changes (Moore et al., 2013).

\section{Local sensing in a mobile patch}

In line with the above, recent observations showing that pheromone sensing elicits unstable zones of polarity are challenging the notion that the polarity patch is established downstream of gradient decoding: wild-type yeast cells - both $S$. cerevisiae and $S$. pombeform a motile polarity patch for chemotropism. From $S$. pombe experiments, my lab described that cells exposed to homogenous fields of low pheromone concentrations exhibit a motile patch that contains active $\mathrm{Cdc} 42$ GTPase and its regulators and effectors. The patch assembles and disassembles at the plasma membrane with a lifetime of less than one to several minutes, exploring most of the cell periphery (Fig. 2) (Bendezú and Martin, 2013). Interestingly, patch dynamics depend on pheromone concentration, with higher concentrations extending the patch residence time. Importantly, during early mating, cells also form an unstable patch before stabilizing a site of growth towards a partner. Subsequent work showed that these patches are fully developed polarity sites, at which secretory vesicles (transporting, among other cargoes, the P-factor) accumulate, and which (in M-cells) are enriched in the $\mathrm{M}$-factor transporter, arguing that pheromones are locally released at these sites. Furthermore, whereas pheromone receptors occupy a large portion of the cell surface, the associated $\mathrm{G} \alpha$ subunit specifically accumulates at the patch, suggesting that the patches are sites of receptor activation, in agreement with the idea that pheromone levels modulate their lifetime (Merlini et al., 2016). Thus, each patch is a mobile communication site, where pheromone signals are sent and locally interpreted. Stimulation by pheromones released at a closely positioned partner cell patch promotes stabilization for chemotropic growth. These observations are captured in a simplified model that reproduces efficient cell pairing in a complex environment (Merlini et al., 2016).

The source of the short lifetime of the patch has not yet been elucidated. Assembly-disassembly dynamics do not require Cdc42 inactivation by GTPase-activating proteins (GAP) (Gallo Castro and Martin, 2018). Instead, it relies on regulation of Ras1, which colocalizes and is active at the polarity patch in $S$. pombe (Merlini et al., 2016, 2018) (Fig. 1). At the patch, Ras1-GTP recruits its sole GAP Gap1, which causes a negative feedback that destabilizes the 


\section{Box 1. Chemotaxis strategies}

Chemotropism can be compared with chemotaxis, which relies on sensing of a graded signal to orient cell movement towards a chemoattractant. For chemotaxis, two conceptually distinct mechanisms - spatial or temporal decoding - have been defined. A spatial decoding system has long been proposed to be used by large cells such as chemotactic eukaryotic amoeba (Cai and Devreotes, 2011; Jin, 2013). Briefly, chemoattractant molecules are detected by receptors that are uniformly present at the plasma membrane and differences in concentration are compared between the front and the back of the cell. Amplification and long-range inhibition mechanisms then magnify the perceived differences to generate a robust axis of polarity. Thus, receptors act at the top of the hierarchy and direct the cytoskeleton re-organization that is necessary for cell migration. More recent work has focused on the excitable properties of the cytoskeletal network and how these are harnessed by the chemotactic signaling system (recently reviewed by Devreotes et al., 2017). Interestingly, chemotactic cells also process information locally at the pseudopod to bias the direction of cell migration (Insall, 2013).

A temporal decoding strategy is used by small cells, such as chemotactic bacteria, like E. coli (Sourjik and Wingreen, 2012). Here, the cell moves and probes its immediate environment at temporal intervals, comparing the results over time. If the sensed chemo-attractant concentration has increased, the cell continues in the same direction. If it has diminished, it randomly tumbles and continues in a new direction. This strategy can be viewed with the opposite hierarchy, where cell movement comes first, with receptor information used to correct for directionality.

\section{Spatial decoding}

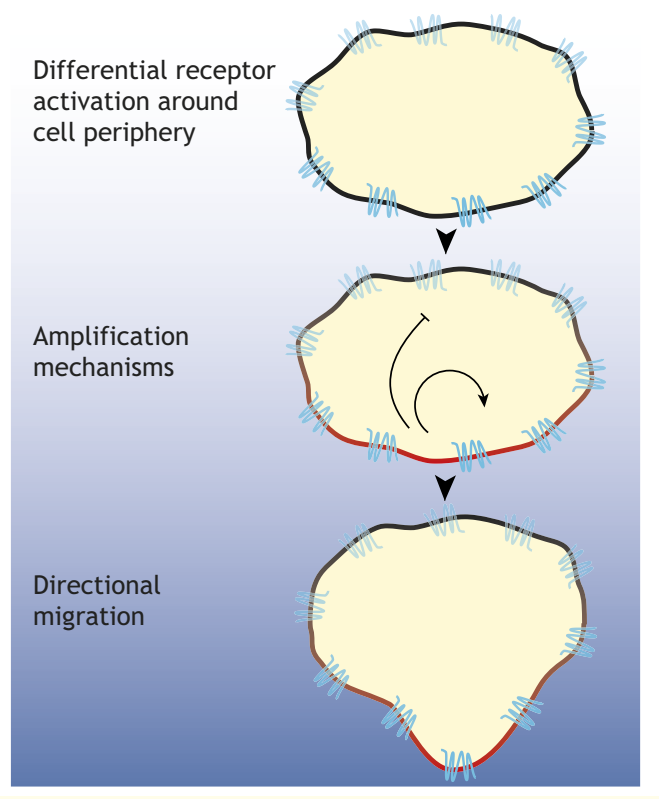

Temporal decoding

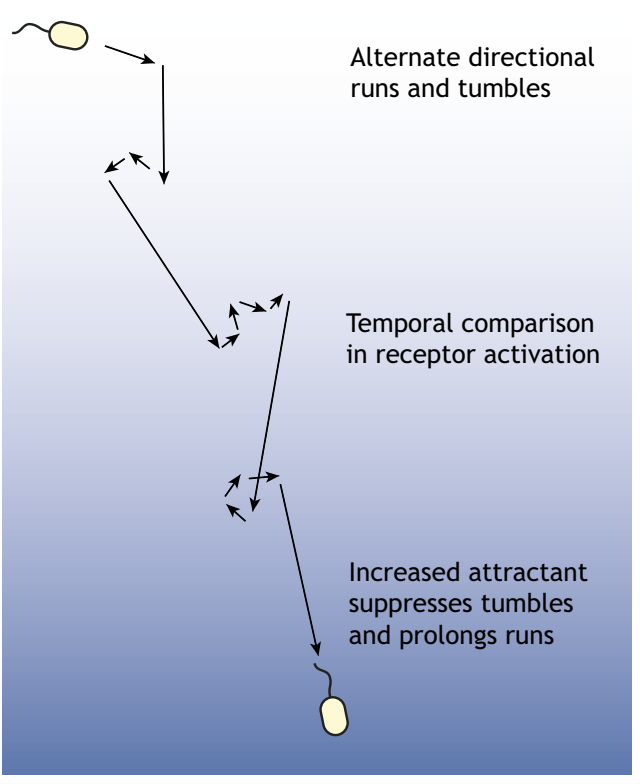

patch (Khalili et al., 2018; Merlini et al., 2018) (Fig. 3). High local pheromone sensing then overcomes this negative control to stabilize the patch. Importantly, because cells lacking the Ras1 GAP retain the ability to form unstable patches when exposed to very low pheromone concentrations, this likely does not represent the sole patch destabilization mechanism.

Similar motile polarity patches underlie chemotropism in $S$. cerevisiae. It had long been known that cells lacking chemotropic information polarize by default at the presumptive bud site (Dorer et al., 1995), and that cells that also lack bud site positioning signals form a motile patch that is unable to sustain polarized growth (Nern and Arkowitz, 2000). Importantly, Lew and colleagues showed that wild-type $S$. cerevisiae cells exposed to homogenous fields of low pheromone concentrations also exhibit a motile Cdc42 patch (Dyer et al., 2013). As in the $S$. pombe situation, higher pheromone levels constrain patch movement, which leads to more pointed growth (Dyer et al., 2013; Hegemann et al., 2015). However, in this case, motility is not achieved by disassembly and re-assembly elsewhere on the cell periphery but by lateral displacement at rates up to $0.01 \mu \mathrm{m}^{2} / \mathrm{min}$ (Fig. 2). Artificial signaling activation through uniform targeting of the MAPK scaffold Ste5 to the plasma membrane (Pryciak and Huntress, 1998) also yields wandering patches that are constrained by high pheromone concentrations (McClure et al., 2015). The source of the motion is proposed to depend on the influx of secretory vesicles along actin cables nucleated at the patch (Fig. 3). As the patch is maintained by positive-feedback systems that have been well characterized during mitotic growth (reviewed in Martin, 2015), local dilution leads to patch lateral displacement. In turn, high pheromone levels counteract the vesicle-dependent dilution effect by activating newly delivered receptors to bias $\mathrm{Cdc} 42$ activation at their location (see below). Consistent with this view, when the $\mathrm{Cdc} 42 \mathrm{GEF}$ is disconnected from the activated receptor, pheromones fail to constrain the patch, which trails $G \beta \gamma$ in its wake. Although these studies were conducted in homogeneous fields of pheromone, similar mechanisms likely operate during gradient perception. Indeed, cells encountering natural gradients produced by partner cells, or placed in engineered gradients in microfluidics chambers, also display a wandering patch, which serves to correct an initial position largely independent of gradient direction, likely at the presumptive bud site (Hegemann et al., 2015). Patch wandering and stabilization at higher pheromone levels are also consistent with the observation that the growth direction in pheromone gradients is gradually improved over time (Paliwal et al., 2007; Segall, 1993). I note that, whereas the modes of patch motility in $S$. cerevisiae and $S$. pombe - lateral displacement versus assembly-disassembly - may at first appear quite distinct, recent studies indicate that both types of movements may be used by both 
Biased random walk

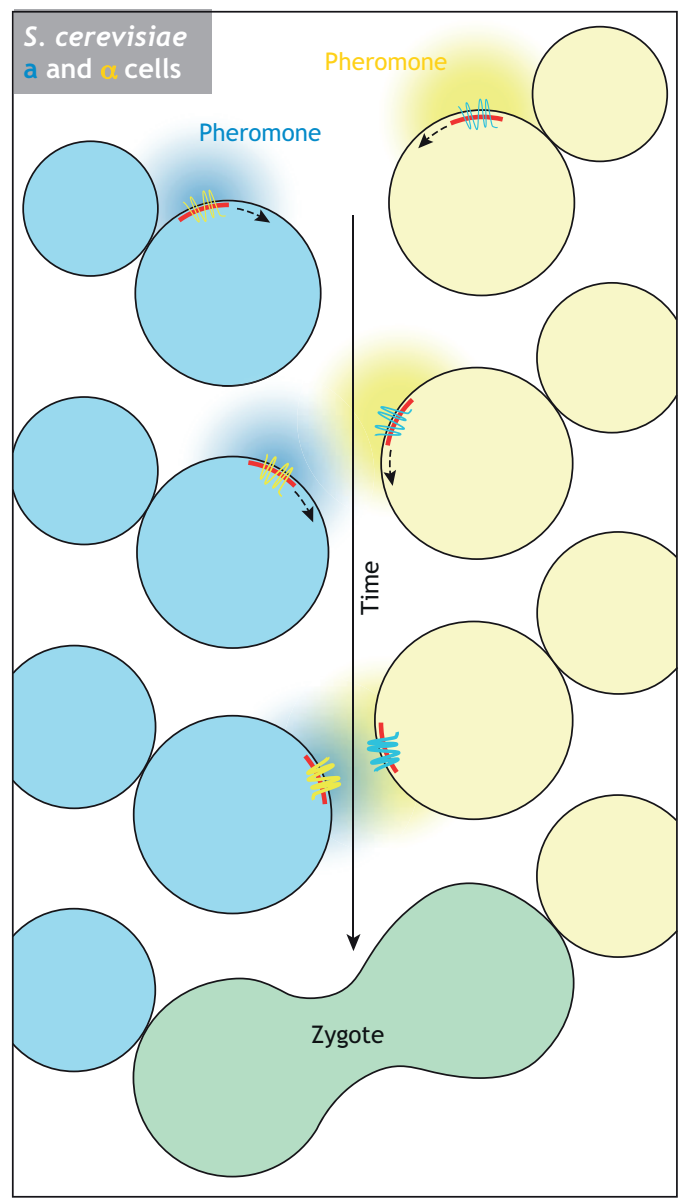

\section{Assembly-disassembly}

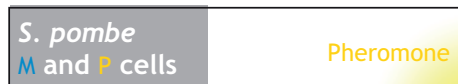

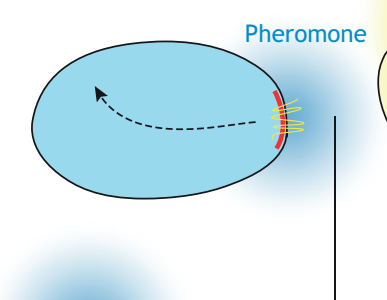
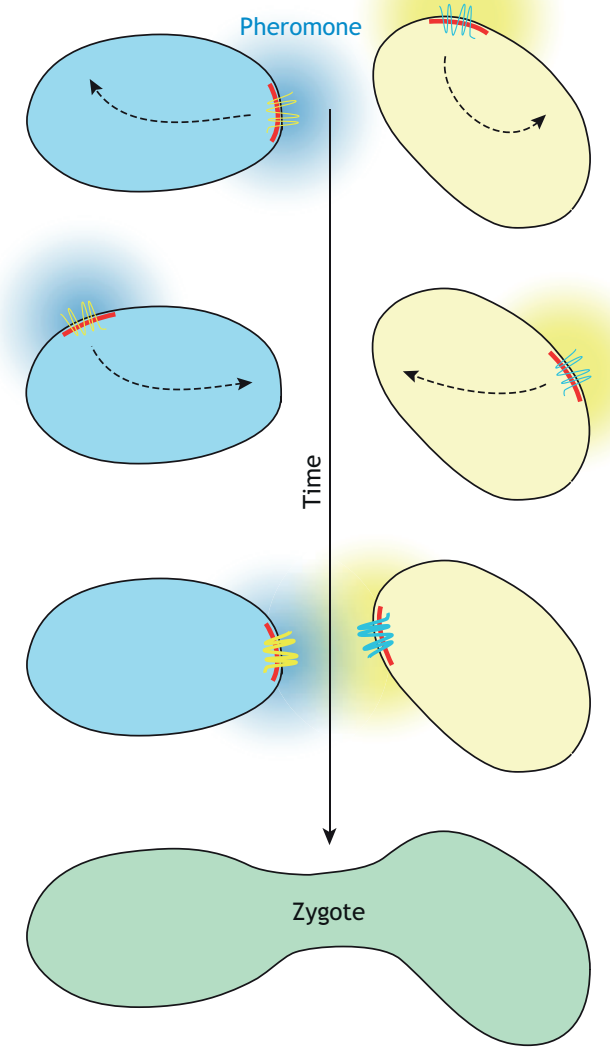

Key W/W W/A Pheromone receptors Polarity patch

Fig. 2. Schematic representation of yeast strategies for chemotropic growth in response to pheromone gradients. A polarity patch (red line) that serves for local pheromone release and sensing is established independently of gradient sensing. The patch is unique and exhibits either assembly-disassembly dynamics (primarily in S. pombe) or lateral displacement (biased random walk, primarily in S. cerevisiae). It forms a communication zone that secretes and responds to pheromones. Higher local pheromone perception leads to patch stabilization, polarized cell growth and cell fusion. Note that the initial distribution of pheromone receptors is not depicted. Blue and yellow indicate different mating types.

organisms. Indeed, uniform $1 \mathrm{nM}$ pheromone concentration also leads to unstable polarization in $S$. cerevisiae, which is very similar to the assembly-disassembly mode of $S$. pombe (Muller et al., 2016); in addition, $S$. pombe cells that lack one of three Cdc42 GAPs displayed notable instances of local biased random walk (Gallo Castro and Martin, 2018).

Collectively, these studies invite a revised model of yeast chemotropism, where a polarity patch is established independently of gradient sensing. The patch is then re-oriented up-gradient by mechanisms involving its destabilization and specific stabilization upon higher local pheromone perception. In this view, the cell does not directly perceive a concentration difference between the front and back, but establishes a specialized cortical zone that serves to locally exchange information: it signals its presence through pheromone release and probes the concentration of the pheromone secreted by the partner cell to decide between staying (when high concentration is sensed) and going (in case of low concentration). This mechanism is more akin to the temporal decoding mechanism used by chemotactic bacteria, yet the sensing and moving entity is not the cell but the polarity patch (see Box 1). One important difference, however, is that the yeast patch may not operate a true temporal comparison by retaining a memory of previous perception, as is the case during bacterial chemotaxis. Although this - to my knowledge - has not been directly tested, studies in homogeneous pheromone concentrations have not reported an adaptation in the patch behavior over time. The cell might, however, measure fractional gradient values rather than absolute gradient concentration (Paliwal et al., 2007). A possible mechanism that remains to be investigated may involve differential sensing of pheromone concentrations within a single patch to skew patch movement towards the gradient source.

\section{Mechanisms of patch stabilization upon pheromone sensing}

A critical question is how pheromone sensing stabilizes the polarity patch. The receptor, initially homogeneously distributed at the plasma membrane, is rapidly internalized upon pheromone exposure and subsequently accumulates at the shmoo tip (Jackson et al., 1991). Whereas this would be problematic for spatial decoding, it is likely advantageous for local sensing. There are diverging views on how receptor polarization is achieved. In S. cerevisiae, it is well established that activation of the receptor by pheromone triggers its phosphorylation by casein kinases I (Yck1 and Yck2) as well as its ubiquitylation, which serve as signal for internalization (reviewed in 

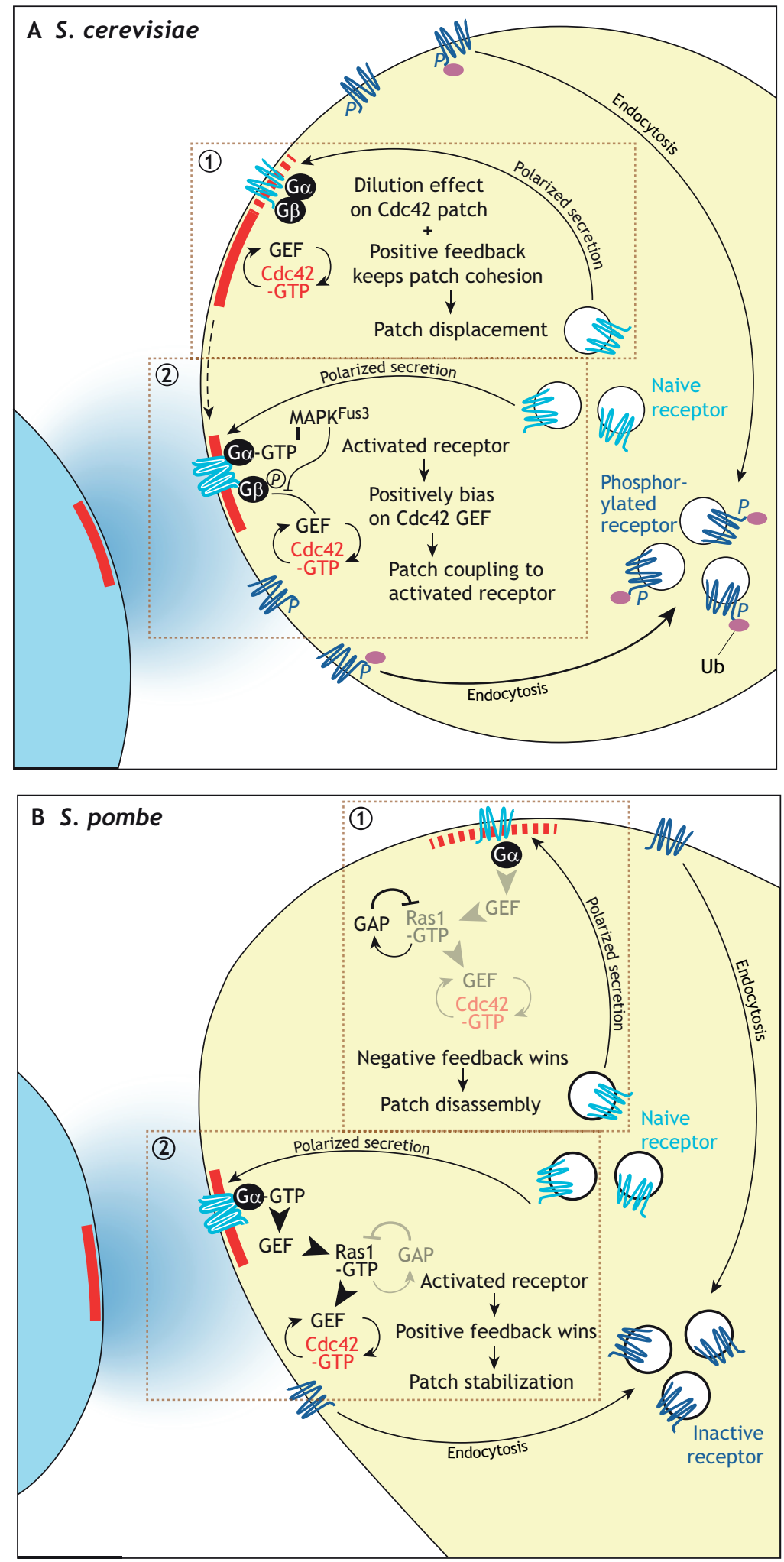

Fig. 3. Molecular model of polarity patch dynamics and stabilization by pheromone sensing. (A) (1) In S. cerevisiae, local secretion is proposed to dilute patch components (red line). As patch integrity is reinforced by positive feedback, this leads to patch lateral displacement. (2) Activation of naïve, unphosphorylated receptor (represented by the light blue and white receptor trace) delivered at the patch leads to $\mathrm{G} \alpha$ activation and $\mathrm{G} \beta$ dissociation, allowing $\mathrm{G} \beta$ to promote $\mathrm{Cdc} 42$ GEF activity. $\mathrm{G} \alpha$ also promotes $\mathrm{G} \beta$ phosphorylation to ensure the interaction with $\mathrm{Cdc} 42$ GEF is short-lived, thus coupling it to the active receptor. The receptor is rapidly phosphorylated $(P)$, leading to its inactivation (darker blue shade), ubiquitylation (pink oval) and internalization. (B) (1) In S. pombe, patch destabilization is promoted by negative control on Ras GTPase, which is caused by GAP recruitment by activated Ras, and other unknown mechanisms. (2) In analogy to the case in $S$. cerevisiae, a pool of naïve receptors is required at the patch to couple pheromone concentration to patch dynamics. Higher pheromone sensing promotes $\mathrm{G} \alpha$ activation, which leads to enhanced Ras GEF activation, thus overcoming the negative control by the Ras GAP.
Alvaro and Thorner, 2016). Because the localization of the receptor to the shmoo tip does not require actin-based transport, but requires its endocytic removal, Suchkov et al. (2010) proposed that receptor polarization is achieved by a mechanism blocking receptor internalization at the cell front. However, recent investigations using fluorogen-activating protein (FAP) tagging to specifically visualize the fate of surface-localized receptor concluded that no region of the plasma membrane is immune to internalization (Emmerstorfer-Augustin et al., 2018). This suggests that receptor accumulation at the shmoo tip does not rely on protection from endocytosis but on de novo production and secretion, which may be polarized independently from actin-based transport as observed in other instances (Bendezú and Martin, 2011; Yamamoto et al., 2010). By extension, naïve receptors are likely delivered to the motile patch. 
Unlike older receptors, naïve receptors are not phosphorylated. Receptor phosphorylation plays two functions: it marks receptors for ubiquitylation and internalization, and also plays an independent role in chemotropism. Indeed, blocking internalization prevents receptor clearing from the surface for polarization (Suchkov et al., 2010), but does not interfere with polarity patch motility (McClure et al., 2015). Such cells also exhibit only modest defects in chemotropic orientation and mating (Ismael et al., 2016). Interestingly, these cells retain a polarized pool of unphosphorylated receptors, which are marked by Sst2, the GTPase-activating protein for G $\alpha$ (Ballon et al., 2006; Suchkov et al., 2010). By contrast, when phosphorylation of the receptor is either blocked or constitutively mimicked, pheromone signaling occurs, but chemotropic growth is largely compromised and cells extend long curved shmoos, which are suggestive of persistent patch wandering (Ismael et al., 2016). Similarly, S. pombe cells with a truncated receptor that lacks its Cterminal tail (and thus can neither be endocytosed nor phosphorylated) sense pheromones (Hirota et al., 2001) but completely fail to stabilize the polarity patch in the later stages of mating, thus blocking cell fusion (see below; Dudin et al., 2016). A model that accounts for the observations is that phosphorylation modifies older receptors to restrict the unphosphorylated pool to the active secretion site. This in turn may create a local differential in pheromone signaling. There is indeed some evidence that phosphorylation (by casein kinases or other kinases) reduces receptor activity though mechanisms distinct from internalization (Chen and Konopka, 1996; Kim et al., 2012). Thus, a transient local pool of naïve, unphosphorylated receptors is critical for the cells to update their pheromone sensing at the patch (Fig. 3).

How does this active pool of receptors transduce pheromone concentration information to the polarity patch? G $\beta \gamma$ is widely considered the critical signaling molecule in $S$. cerevisiae. Like Sst2, G $\beta$ is polarized even in absence of receptor endocytosis and independently of its association with the Cdc42 GEF (McClure et al., 2015), suggesting that it concentrates at sites of unphosphorylated receptor activation. Interestingly, G $\beta$ is itself phosphorylated (Cole and Reed, 1991) and this phosphorylation is required to stabilize the polarity site (Deflorio et al., 2013). One model is that phosphorylation of $\mathrm{G} \beta$ weakens its interaction with the Cdc42 GEF, allowing for more frequent probing of activated receptors, to update on local pheromone concentration information and prevent polarity site drift from active receptors (Deflorio et al., 2013) (Fig. 3). Phosphorylation of G $\beta$ depends on G $\alpha$ (Deflorio et al., 2013; Li et al., 1998), which is required for G $\beta$ polarization and for polarity patch constraint (McClure et al., 2015). Interestingly, G $\alpha$ binds $\mathrm{G} \beta$ through two interfaces, one of which is critical for its chemotropic role (Strickfaden and Pryciak, 2008). The relevant kinase may be the MAPK Fus3, as G $\alpha$ directly binds Fus3, and this interaction promotes chemotropism (Metodiev et al., 2002). The MAPK also plays a second positive role in patch stabilization by promoting the nuclear export of the Cdc42 GEF (Hegemann et al., 2015). However, one study found that Fus3 is dispensable for chemotropism (Strickfaden and Pryciak, 2008). In summary, G $\alpha$ activation may not only free G $\beta \gamma$ to bind the Cdc42 GEF, but also promote its phosphorylation through Fus3 or another kinase to ensure the interaction is short lived.

$\mathrm{G} \alpha$ and $\mathrm{G} \beta$ may influence chemotropism by additional means. For instance, $\mathrm{G} \beta$ phosphorylation permits its monoubiquitylation, a modification that also influences its chemotropic function (Zhu et al., 2011). Another possible mean of control may be through Go ubiquitylation (Wang et al., 2005), which is required for a normal morphogenetic response and controls its internalization and degradation through a mechanism partly distinct from receptor

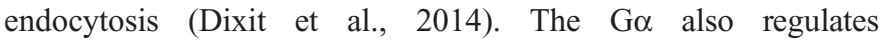
chemotropism positively - perhaps to enhance morphogenesis by binding the RNA-binding protein Scp160, a cortical ER protein that promotes the transport of mRNA-encoding polarity and secretion factors along actin cables (Gelin-Licht et al., 2012; Guo et al., 2003). These positive functions of the $\mathrm{G} \alpha$ are intriguing in light of the pathway wiring in $S$. pombe, where $\mathrm{G} \alpha$ is the sole signal transducer, and promotes localization and/or activation of the Ras GEF through unknown molecular connections (Fig. 3).

In summary, unphosphorylated receptors that are delivered at the mobile patch locally probe pheromones. Activated $\mathrm{G} \alpha$ frees and promotes the phosphorylation of G $\beta$, which transiently binds the $\mathrm{Cdc} 42 \mathrm{GEF}$, thus countering patch movement by coupling $\mathrm{Cdc} 42$ activity with the activated receptor. An interesting question is whether this mechanism suffices to fully explain chemotropism, or whether there is also a contribution from receptor sensing away from the patch. A computational model from Stone and colleagues, based on the finding that $\mathrm{G} \beta$ phosphorylation also increases its interaction with the casein kinases that phosphorylate the pheromone receptor, yielded receptor polarization to the cell front without invoking Cdc42 (Ismael et al., 2016). The idea is that the interaction titrates the kinases away from the receptor, creating a local feedback that delays receptor phosphorylation to enhance its activity and inhibit endocytosis. This opens the possibility of a Cdc42-independent mode of anisotropy in activated receptors, which may perhaps work at a short-scale within a patch. However, whether receptor polarization can take place independently of $\mathrm{Cdc} 42$ in cells remains to be tested. An intriguing observation is that, although the initial patch position is often not aligned with the pheromone gradient, it may not be completely random relative to the pheromone gradient, whether the gradient is generated artificially (Hegemann et al., 2015; 59\% of cells polarized towards the gradient source) or by a mating partner (Henderson et al., 2018 preprint). It will be important in the future to establish whether and how cells can also use some elements of spatial decoding to bias the initial position of the patch.

\section{From growth to fusion}

Fungal cells are protected by a cell wall, which is locally remodeled during polarized growth and protects cellular integrity (Davì and Minc, 2015). Indeed, cell growth is powered by the internal turgor pressure, which pushes against the resisting cell wall. Local wall remodeling allows the uniform internal pressure to expand the cell locally to achieve polarized growth. Although this process is not understood in great detail, it relies on polarized secretion under control of $\mathrm{Cdc} 42$, which leads to local delivery of transmembrane cell wall synthases and secretion of hydrolases to locally promote cell wall expansion (Martin and Arkowitz, 2014). During mating, as cells polarize towards each other, they extend a growth projection. Importantly, during polarized growth, cell wall integrity is almost never compromised, which would result in cell lysis. How is this behavior changed to induce the formation of a hole in the cell wall in preparation for fusion?

Cell wall digestion is thought to rely on a local increase in the concentration and/or activity of secreted hydrolases (Fig. 4). Cell contact can passively help this increase by confining secreted enzymes to the space between the two partner cells (Huberman and Murray, 2014). More importantly, the cells actively re-organize and confine their secretion zone, enhancing their polarized state. An early electron microscopy study of $S$. cerevisiae mating described a cluster of vesicles that requires the function of Spa2, a protein 


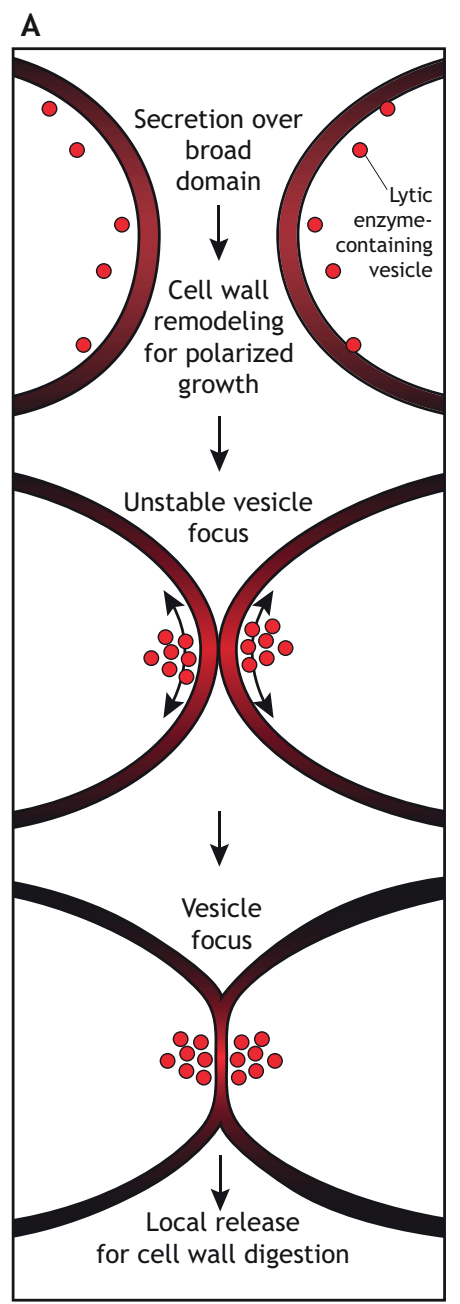

\section{B S. cerevisiae}

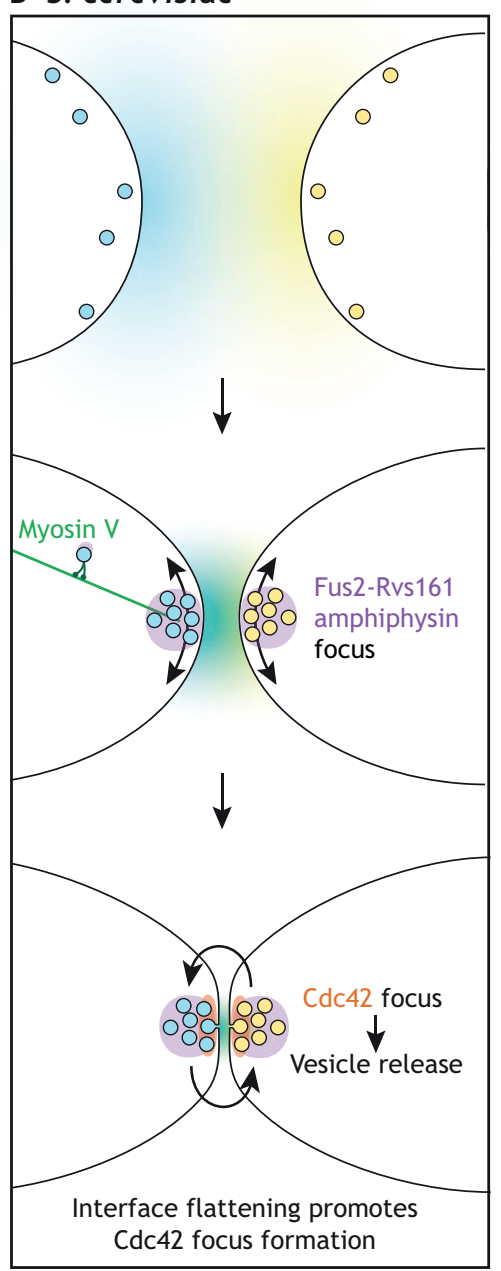

\section{S. pombe}

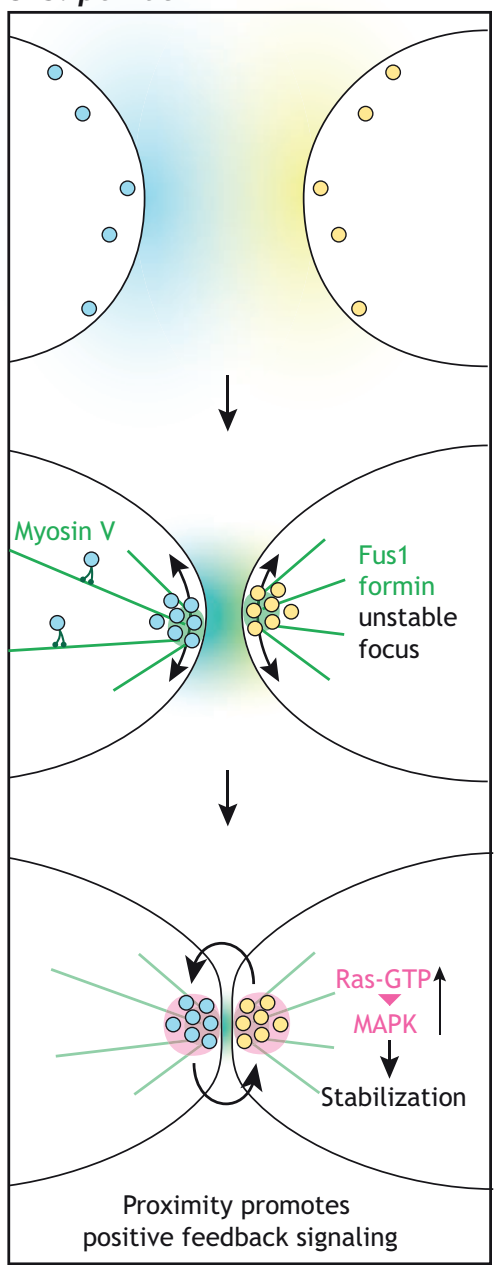

Fig. 4. Strategies for coupling cell wall digestion with cell-cell contact. (A) Cell wall lytic enzymes (red) are secreted by polarized exocytosis. These are required for cell wall remodeling for expansion during polarized growth. During mating, the secretory vesicles are concentrated over a small surface area. If the site of secretion is not fixed and moves over time, the cell wall keeps expanding. If the site of secretion is fixed, this leads to a local increase in secreted enzyme concentration and cell wall digestion, which needs to be coordinated with cell-cell contact to avoid lysis. (B) In S. cerevisiae, a focus made of vesicles and a Fus2-Rvs161 amphiphysin-like complex is also formed by means that may involve the actin cytoskeleton (green line), like in S. pombe. Flattening of the plasma membrane curvature, which occurs as cells grow against each other, is proposed to induce a conformational change in the Fus2-Rvs161 complex. This allows the recruitment of $\mathrm{Cdc} 42$, which leads to local release of the vesicular pool. In the middle and right panels, yellow and blue indicate pheromones. (C) In S. pombe, the fusion focus, an actin aster nucleated by the formin Fus1, promotes the concentration of vesicles delivered by type $V$ myosin. The focus is initially unstable, leading to secretion of lytic enzymes over a broad zone and cell growth. As the focus is also a site of pheromone release and perception, the increasing proximity between the two cells enhances the positive feedback signaling between the two partners. This local increase in MAPK signaling leads to focus stabilization, driving cell wall digestion. Lipidated pheromones (a- and M-factors) are secreted from the cytosol by a dedicated ABC-type transporter, itself delivered by polarized secretion (not shown in the figure).

associated with the actin polymerization factor formin, suggesting an important role of the actin cytoskeleton (Gammie et al., 1998). Studies in $S$. pombe established the importance of the actin cytoskeleton in organizing this vesicle cluster, which is also observed in electron micrographs of cell pairs in this organism (unpublished observations from my laboratory). A formin that is specifically expressed during mating, Fus1, is essential for cell wall digestion (Petersen et al., 1998, 1995). It assembles a specialized actin structure at the site of cell-cell contact, called the fusion focus, which resembles an aster of linear actin filaments that drive the concentration of type $\mathrm{V}$ myosin motors and their cargoes to a focused cortical zone (Dudin et al., 2015). Several other actin-binding proteins, such as tropomyosin and its associated factors, help tighten the focus (Dudin et al., 2017; Kurahashi et al., 2002). This results in the delivery of vesicles carrying cell wall hydrolases to a confined zone. Indeed, hydrolase cargoes and vesicle-associated exocyst complex subunits concentrate at the site of fusion (Dudin et al., 2015; Sharifmoghadam et al., 2010). Because cell wall synthases remain more broadly distributed at the contact site, my group proposed this leads to an imbalance between synthetic and lytic reactions in the cell wall, promoting local digestion (Dudin et al., 2015; Martin, 2016).

A critical question is what controls the shift between cell wall expansion and digestion to avoid lysis. We modified $S$. pombe cells to act as autocrine cells by expressing the receptor for the self-produced pheromone and discovered that these mount a fusion response in absence of a partner cell, and thus lyse (Dudin et al., 2016). This is due to a positive feedback where the fusion focus localizes the pheromone signaling pathway (pheromone transporter, receptors and MAPK cascade), which in turn promotes focus 
stabilization: local MAPK activity stabilizes the focus, which concentrates pheromone release and leads to enhanced receptorRas-MAPK signaling. This further stabilizes the focus, and so on. This self-enhancing system normally occurs between two partner cells, each stabilizing the focus of the partner, similar to the case of cell pairing during chemotropism. Inhibition of Ras 1 activity by its GAP restrains the system and restricts focus stabilization, and thus cell wall digestion, to cells in close proximity (Merlini et al., 2018). Interestingly, whereas local MAPK recruitment stabilizes the polarity site and triggers cell wall digestion, a constitutively active MAPK present ubiquitously at the membrane blocked cell fusion, indicating a critical role for a spatially restricted MAPK signal (Dudin et al., 2016). In this view, conversion from a process of cell wall expansion to local digestion is a natural consequence of the increasing proximity of the two partner cells, which leads the positive pheromone-dependent focusing feedback to overcome negative Ras1-GAP controls (Fig. 4).

In $S$. cerevisiae, important work from the laboratory of Mark Rose has concentrated on the function of the Fus 2 protein, which is required for efficient fusion. Fus2 forms a focus at the shmoo tip, but is not itself required for vesicle clustering (Gammie et al., 1998; Paterson et al., 2008). Fus2, which has both an amphiphysin-like and a Dbl homology (DH) domain, associates with the BAR-domain-containing protein Rvs161, with which it is transported to the shmoo tip by myosin V (Brizzio et al., 1998; Paterson et al., 2008; Sheltzer and Rose, 2009). This likely forms an amphiphysin-like complex proposed to bind vesicles, though it does not visibly associate with Sec4-decorated secretory vesicles (Sheltzer and Rose, 2009; Stein et al., 2015). The function of Rvs161 in this complex is distinct from its role in shaping endocytic vesicles with its alternative binding partner Rvs167 (Brizzio et al., 1998). Interestingly, the Fus2 DH domain does not appear to function as a GEF, but binds the GTP-loaded form of Cdc42 through an interaction involving the Cdc42 Rho-insert domain (Ydenberg et al., 2012). This interaction is specifically important for cell fusion: indeed, $\mathrm{Cdc} 42$ mutants that are deficient in this interaction are competent for cell polarization, but exhibit fusion defects. Furthermore, this interaction underlies a focal concentration of $\mathrm{Cdc} 42$, which does not occur in shmoos, but specifically at the pre-fusion zone (Smith et al., 2017). As this localization also depends on the overall membrane curvature at the shmoo tip, which becomes flatter as cells pair together, the proposed model is that the Fus2-Rvs161 complex recognizes the modification in membrane curvature through a conformational change that induces $\mathrm{Cdc} 42$ binding. Cdc42 focusing may in turn promote local exocytosis of pre-accumulated vesicles at this point for cell wall digestion. Thus, the conversion from cell wall expansion to digestion would be a physical consequence of cell pair formation, where growth of the cells against each other leads to interface flattening (Fig. 4).

Although studies in the two yeast models have investigated largely distinct molecular components, we are now starting to see points of convergence, in particular in the formation of a focus at the site of cell contact, which can be viewed as a hyperpolarization response. An actin focus may exist in $S$. cerevisiae, especially given the reported roles of formin, tropomyosin and myosin $\mathrm{V}$ in cell fusion (reviewed in Merlini et al., 2013). There is also increasing evidence for a role of MAPK signaling for fusion, like in $S$. pombe. Indeed, cells with loss-of-function alleles of the MAPK fus 3 display specific fusion deficiency (Elion et al., 1990; Fujimura, 1994), and a recent study suggested an increase in MAPK activity just before cell fusion (Conlon et al., 2016). As Fus3 localizes to the shmoo tip in a manner dependent on interaction with its scaffold Ste5 and substrates (Conlon et al., 2016; Maeder et al., 2007; van Drogen et al., 2001), it is likely that it acts locally at the fusion site. A role for MAPK in fusion was also recently proposed during somatic cell fusion in Neurospora (see Box 2). Reciprocally, mutants in the $S$. pombe Rvs161 homolog Hob3 displays a fusion defect and may

\section{Box 2. Somatic chemotropism and fusion in Neurospora crassa}

In addition to cell fusion during sexual development, filamentous fungi also display fusion between somatic hyphae, a process called anastomosis. The chemotropic signal inducing growth and fusion during anastomosis remains to be defined, but is believed to be identical in the two partners. The partner cells resolve this problem through a ping-pong mechanism in which they coordinately alternate between states of signal release and perception (Fleissner et al., 2009b). Interestingly, this ping-pong mechanism involves two MAPK pathways. As one cell accumulates the homolog of the yeast pheromoneresponsive MAPK, mitogen-activated protein kinase 2 (MAK-2), and its scaffold, HAM-5, the other accumulates the WD40 protein SO (SOFT), proposed to act as scaffold for the homolog of the yeast cell wall integrity MAPK MAK-1, which visibly accumulate only at fusion time (Fleissner et al., 2009b; Jonkers et al., 2014; Weichert et al., 2016). MAK-2 induces dispersion of SO, which in consequence oscillates in alternate phase. The periodic oscillations are proposed to stem from the pulsatile nature of an activator, possibly MAK-2, that induces vesicle docking and prevents their fusion until its activity diminishes (Goryachev et al., 2012).

There are many interesting analogies with the yeast chemotropic and fusion mechanisms. For instance, oscillatory signaling shows reinforcement as cells grow towards each other, resulting in progressive concentration of MAK-2 at cell tips (Fleissner et al., 2009b). The small GTPases CDC-42 and RAC-1, which are present and active at the growing tip, are required for chemotropic growth, MAK-2 recruitment and its oscillatory dynamics (Lichius et al., 2014). Sterol intermediates cause fusion defects by interfering with MAK-1 localization (Weichert et al., 2016). MAK-2 is also required for the final stages of cell fusion leading to cytoplasmic mixing (Serrano et al., 2018). Numerous other complexes, such as reactive oxygen species (ROS)-generating dihydronicotinamide-adenine dinucleotide phosphate (NADPH) oxidase and striatin-interacting phosphatase and kinase (STRIPAK) complexes play important roles in Neurospora germling fusion. See Fleissner and Herzog (2016) for a recent review.

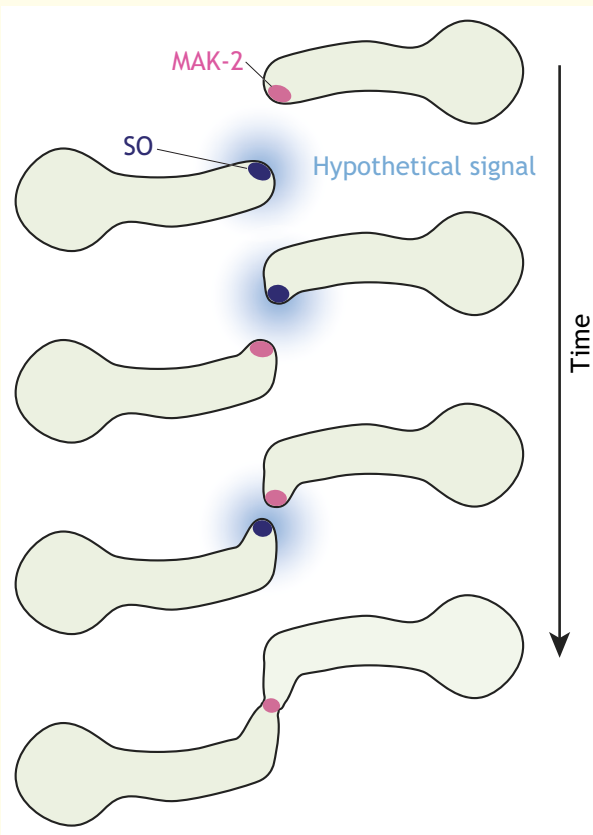


function with a yet-to-be-identified functional homolog of Fus2 (Dudin et al., 2017). Thus, the increased proximity of partner cells as they grow towards each other may induce both meaningful physical and signaling changes, which additively contribute to a successful fusion process. Additional control mechanisms likely also participate. For instance, yeast cells monitor cell wall integrity, and $S$. cerevisiae mutants in cell wall sensors exhibit pheromonedependent lysis (Hall and Rose, 2019; Rajavel et al., 1999). Conversely, mutations enhancing cell wall integrity signaling block pheromone-induced cell death (Philips and Herskowitz, 1997; Zhang et al., 2006). Continued studies in a range of fungal systems should help illuminate how the cell safely pierces its cell wall.

\section{Plasma membrane merging}

The final stage of cell fusion involves merging of the plasma membranes of the partner cells. This is arguably the defining step in cell-cell fusion, which has recently received much attention in a wide range of organisms. Membrane merging requires significant force to overcome the repulsive charge and hydration forces that normally keep biological membranes separate. In well-characterized paradigms of membrane fusion, including vesicle or viral fusion, this force is conferred by fusogens; these membrane proteins are able to release stored energy through complex formation or conformational change that bring membranes in very close contact (Hernández and Podbilewicz, 2017). Excitingly, recent work has uncovered a family of virus-derived fusogens, variously called Hap2, Gcs1 or Eff-1, that mediate cell-cell fusion in a wide range of organisms (Clark, 2018). However, no member of this class of proteins appears to exist in fungi (nor vertebrates), raising the question of what mediates membrane merging in fungi.

Loss-of-function in a fusogen is expected to yield cell pairs that are arrested with a digested cell wall and apposed plasma membranes. However, only a small group of genes exhibit a lossof-function phenotype that fulfill this criterion, the most prominent member being the fungal-specific, and highly conserved, multitransmembrane protein Prm1. Prm1 was initially identified through a bioinformatic strategy in $S$. cerevisiae, where its loss causes $\sim 50 \%$ of cell pairs to fail at fusion, with their plasma membranes remaining at a distance of $\sim 8 \mathrm{~nm}$ (Heiman and Walter, 2000). These cells have successfully digested their cell wall and exhibit a characteristic phenotype in which one cell extends a protrusion inside the other. Studies on other fungi have confirmed the role of Prm1 in fusion: prm1s partner cells in Neurospora, Cryptococcus and $S$. pombe protrude into each other and are partly or almost completely fusion deficient (Curto et al., 2014; Fleissner et al., 2009a; Fu and Heitman, 2017). However, electron micrographs of prm1 $S$. pombe cells did not reveal apposed plasma membranes (Curto et al., 2014), possibly because of efficient cell wall repair mechanisms. The molecular function of Prm1 is not known. In $S$. cerevisiae, it forms homodimers, which are stabilized by disulfide bridges. This promotes the exit of Prm1 from the ER and localization to the plasma membrane at the fusion site where it exerts its function (Engel et al., 2010; Olmo and Grote, 2010a,b). Prm1 has four transmembrane domains and a hydrophobic region on one of its extracellular loops (Olmo and Grote, 2010a), which raises the exciting but untested possibility that it may be able to insert in the partner cell membrane to promote fusion. Work on S. pombe has also suggested Prm1 stimulates fusion by promoting the externalization of phosphatidylserine to the outer plasma membrane leaflet at the point of cell-cell contact (Curto et al., 2014). In addition, because $S$. cerevisiae and Neurospora prm $1 \Delta$ cells (but not $S$. pombe) often lyse, Prm1 also serves a protective function against cell lysis during fusion, which may be also catalyzed by other, as-yet unidentified fusogen(s) (Aguilar et al., 2007; Curto et al., 2014; Jin et al., 2004; Palma-Guerrero et al., 2014).

A second family of four-pass transmembrane proteins, including $S$. cerevisiae Fig1 and $S$. pombe Dni1 and Dni2, appears to have roles in membrane fusion in several fungi. Cells lacking figl, dnil or dni2, like prm $1 \Delta$ cells, exhibit protrusion, membrane apposition and fusion defects (Aguilar et al., 2007; Clemente-Ramos et al., 2009). Because $S$. cerevisiae fig $1 \Delta$ cells also lyse and the lysis and fusion defects are to a large part rescued by addition of $\mathrm{Ca}^{2+}$, Fig1 has been proposed to promote $\mathrm{Ca}^{2+}$ influx (Erdman et al., 1998; Muller et al., 2003). Importantly, addition of $\mathrm{Ca}^{2+}$ also ameliorates the fusion efficiency of prm $1 \Delta$ cells in this organism (Aguilar et al., 2007), suggesting regulation of $\mathrm{Ca}^{2+}$ is critical for fusion. However, $\mathrm{Ca}^{2+}$ does not modulate fusion in S. pombe (Clemente-Ramos et al., 2009), where Dni2 is proposed to function as a claudin-like compartmentalization factor to restrict Dni1 to the fusion site (Curto et al., 2018).

A number of other fungal proteins and factors have been implicated in membrane fusion, as their loss leads to partial cell fusion defects with one cell protruding into the other. In $S$. cerevisiae, these include the Golgi protease Kex2, which was proposed to post-translationally modify a fusion factor acting additively with Prm1 (Heiman et al., 2007). Two enzymes involved in ergosterol biosynthesis, Erg6 and Erg4, promote efficient cell fusion, which suggests a role for sterols, although fusion defects in $\operatorname{erg} 4 \Delta$ were shown to arise because of accumulation of a sterol intermediate, rather than absence of ergosterol (Aguilar et al., 2010; Jin et al., 2008). Furthermore, direct physical interaction between the pheromone receptors on the two partner cells has also been proposed to promote membrane fusion, although mutations abrogating this interaction blocked cell fusion before membrane apposition (Shi et al., 2007). In Neurospora, the transmembrane protein Lfd1 appears to be involved in membrane fusion (PalmaGuerrero et al., 2014). Identification of the fungal fusogen(s) remains a clear challenge for the future.

\section{Conclusions and perspectives}

The recent studies on chemotropism in $S$. cerevisiae and $S$. pombe have brought about a complete reversal in the concepts of gradient sensing. Given their distant evolutionary origin, there are of course important differences in the two yeast models, which in many cases help to highlight diverse realization of the same phenomenon. Instead of globally comparing chemoattractant concentration in its surrounding, the cell assembles a specialized detection patch that sequentially probes the local pheromone concentration. Thus, conceptually, cell polarization functions upstream of gradient detection. The subsequent digestion of the cell wall between two partner cells is a direct consequence of the change in geometry due to the growth of the partners towards each other.

There remain many unanswered questions, especially on the nature and mechanism of the membrane fusogen(s), but also on a possible bias in initial polarization, on the role of local MAPK signaling in patch stabilization, and on the relative contributions of various physical changes for cell wall digestion. The novel concepts of gradient source and detection at a local patch may also prompt re-investigation of old questions, for instance, on the role of pheromone proteases in sharpening gradients or the possible differences between the two fusing cell types, conferred in part by the distinct nature of the two pheromones. Finally, our recent finding that there are active mechanisms to prevent chemotropism and fusion in zygotes (Vjestica et al., 2018) opens questions into 
the regulatory mechanisms that limit fusion to exactly two partners. Active research in these areas promises exciting discoveries in the near future.

\section{Acknowledgements}

I thank members of my laboratory for discussions and Dr Laura Merlini, Dr Aleksandar Vjestica, Dr Gaowen Liu and Ingrid Billault-Chaumartin for comments on the manuscript.

\section{Competing interests}

declare no competing or financial interests.

\section{Funding}

Research in my laboratory is supported by a Schweizerischer Nationalfonds zur Förderung der Wissenschaftlichen Forschung (Swiss National Science Foundation) research grant (310030B_176396) and an European Research Council consolidator grant (CellFusion).

\section{References}

Aguilar, P. S., Engel, A. and Walter, P. (2007). The plasma membrane proteins Prm1 and Fig1 ascertain fidelity of membrane fusion during yeast mating. Mol. Biol. Cell 18, 547-556. doi:10.1091/mbc.e06-09-0776

Aguilar, P. S., Heiman, M. G., Walther, T. C., Engel, A., Schwudke, D., Gushwa, N., Kurzchalia, T. and Walter, P. (2010). Structure of sterol aliphatic chains affects yeast cell shape and cell fusion during mating. Proc. Natl. Acad. Sci. USA 107, 4170-4175. doi:10.1073/pnas.0914094107

Alvaro, C. G. and Thorner, J. (2016). Heterotrimeric G protein-coupled receptor signaling in yeast mating pheromone response. J. Biol. Chem. 291, 7788-7795. doi:10.1074/jbc.R116.714980

Arkowitz, R. A. (2009). Chemical gradients and chemotropism in yeast. Cold Spring Harb. Perspect. Biol. 1, a001958. doi:10.1101/cshperspect.a001958

Artemenko, Y., Lampert, T. J. and Devreotes, P. N. (2014). Moving towards a paradigm: common mechanisms of chemotactic signaling in Dictyostelium and mammalian leukocytes. Cell. Mol. Life Sci. 71, 3711-3747. doi:10.1007/s00018014-1638-8

Ballon, D. R., Flanary, P. L., Gladue, D. P., Konopka, J. B., Dohlman, H. G. and Thorner, J. (2006). DEP-domain-mediated regulation of GPCR signaling responses. Cell 126, 1079-1093. doi:10.1016/j.cell.2006.07.030

Bendezú, F. O. and Martin, S. G. (2011). Actin cables and the exocyst form two independent morphogenesis pathways in the fission yeast. Mol. Biol. Cell 22 44-53. doi:10.1091/mbc.e10-08-0720

Bendezú, F. O. and Martin, S. G. (2013). Cdc42 explores the cell periphery for mate selection in fission yeast. Curr. Biol. 23, 42-47. doi:10.1016/j.cub.2012.10.042

Brizzio, V., Gammie, A. E. and Rose, M. D. (1998). Rvs161p interacts with Fus2p to promote cell fusion in Saccharomyces cerevisiae. J. Cell Biol. 141, 567-584 doi:10.1083/jcb.141.3.567

Butty, A. C., Pryciak, P. M., Huang, L. S., Herskowitz, I. and Peter, M. (1998). The role of Far1p in linking the heterotrimeric $\mathrm{G}$ protein to polarity establishment proteins during yeast mating. Science 282, 1511-1516. doi:10.1126/science.282. 5393.1511

Cai, H. and Devreotes, P. N. (2011). Moving in the right direction: how eukaryotic cells migrate along chemical gradients. Semin. Cell Dev. Biol. 22, 834-841. doi:10. 1016/j.semcdb.2011.07.020

Chen, Q. and Konopka, J. B. (1996). Regulation of the G-protein-coupled alphafactor pheromone receptor by phosphorylation. Mol. Cell. Biol. 16, 247-257. doi:10.1128/MCB.16.1.247

Clark, T. (2018). HAP2/GCS1: Mounting evidence of our true biological EVE? PLoS Biol. 16, e3000007. doi:10.1371/journal.pbio. 3000007

Clemente-Ramos, J. A., Martín-García, R., Sharifmoghadam, M. R., Konomi, M., Osumi, M. and Valdivieso, M.-H. (2009). The tetraspan protein Dni1p is required for correct membrane organization and cell wall remodelling during mating in Schizosaccharomyces pombe. Mol. Microbiol. 73, 695-709. doi:10. $1111 / j .1365-2958.2009 .06800 . x$

Cole, G. M. and Reed, S. I. (1991). Pheromone-induced phosphorylation of a G protein beta subunit in $\mathrm{S}$. cerevisiae is associated with an adaptive response to mating pheromone. Cell 64, 703-716. doi:10.1016/0092-8674(91)90500-X

Conlon, P., Gelin-Licht, R., Ganesan, A., Zhang, J. and Levchenko, A. (2016). Single-cell dynamics and variability of MAPK activity in a yeast differentiation pathway. Proc. Natl. Acad. Sci. USA 113, E5896-E5905. doi:10.1073/pnas. 1610081113

Curto, M.-A., Sharifmoghadam, M. R., Calpena, E., De León, N., Hoya, M., Doncel, C., Leatherwood, J. and Valdivieso, M.-H. (2014). Membrane organization and cell fusion during mating in fission yeast requires multi-pass membrane protein Prm1. Genetics 196, 1059-1076. doi:10.1534/genetics.113. 159558

Curto, M.-A., Moro, S., Yanguas, F., Gutiérrez-González, C. and Valdivieso, M.-H. (2018). The ancient claudin Dni2 facilitates yeast cell fusion by compartmentalizing Dni1 into a membrane subdomain. Cell. Mol. Life Sci. $\mathbf{7 5}$, 1687-1706. doi:10.1007/s00018-017-2709-4

Davì, V. and Minc, N. (2015). Mechanics and morphogenesis of fission yeast cells. Curr. Opin. Microbiol. 28, 36-45. doi:10.1016/j.mib.2015.07.010

Deflorio, R., Brett, M.-E., Waszczak, N., Apollinari, E., Metodiev, M. V., Dubrovskyi, O., Eddington, D., Arkowitz, R. A. and Stone, D. E. (2013) Phosphorylation of Gbeta is crucial for efficient chemotropism in yeast. J. Cell Sci. 126, 2997-3009. doi:10.1242/jcs.112797

Devreotes, P. N., Bhattacharya, S., Edwards, M., Iglesias, P. A., Lampert, T. and Miao, Y. (2017). Excitable signal transduction networks in directed cell migration. Annu. Rev. Cell Dev. Biol. 33, 103-125. doi:10.1146/annurev-cellbio-100616-060739

Dixit, G., Baker, R., Sacks, C. M., Torres, M. P. and Dohlman, H. G. (2014) Guanine nucleotide-binding protein (Galpha) endocytosis by a cascade of ubiquitin binding domain proteins is required for sustained morphogenesis and proper mating in yeast. J. Biol. Chem. 289, 15052-15063. doi:10.1074/jbc.M114. 566117

Dorer, R., Pryciak, P. M. and Hartwell, L. H. (1995). Saccharomyces cerevisiae cells execute a default pathway to select a mate in the absence of pheromone gradients. J. Cell Biol. 131, 845-861. doi:10.1083/jcb.131.4.845

Dudin, O., Bendezú, F. O., Groux, R., Laroche, T., Seitz, A. and Martin, S. G. (2015). A formin-nucleated actin aster concentrates cell wall hydrolases for cell fusion in fission yeast. J. Cell Biol. 208, 897-911. doi:10.1083/jcb.201411124

Dudin, O., Merlini, L. and Martin, S. G. (2016). Spatial focalization of pheromone/ MAPK signaling triggers commitment to cell-cell fusion. Genes Dev. 30 , 2226-2239. doi:10.1101/gad.286922.116

Dudin, O., Merlini, L., Bendezú, F. O., Groux, R., Vincenzetti, V. and Martin, S. G. (2017). A systematic screen for morphological abnormalities during fission yeast sexual reproduction identifies a mechanism of actin aster formation for cell fusion. PLoS Genet. 13, e1006721. doi:10.1371/journal.pgen.1006721

Dyer, J. M., Savage, N. S., Jin, M., Zyla, T. R., Elston, T. C. and Lew, D. J. (2013) Tracking shallow chemical gradients by actin-driven wandering of the polarization site. Curr. Biol. 23, 32-41. doi:10.1016/j.cub.2012.11.014

Elion, E. A., Grisafi, P. L. and Fink, G. R. (1990). FUS3 encodes a cdc2+/CDC28related kinase required for the transition from mitosis into conjugation. Cell $\mathbf{6 0}$ 649-664. doi:10.1016/0092-8674(90)90668-5

Emmerstorfer-Augustin, A., Augustin, C. M., Shams, S. and Thorner, J. (2018). Tracking yeast pheromone receptor Ste2 endocytosis using fluorogen-activating protein (FAP) tagging. Mol. Biol. Cell 29, 2603-2799. doi:10.1091/mbc.E18-07-0424

Engel, A., Aguilar, P. S. and Walter, P. (2010). The yeast cell fusion protein Prm1p requires covalent dimerization to promote membrane fusion. PLOS ONE 5 e10593. doi:10.1371/journal.pone.0010593

Erdman, S., Lin, L., Malczynski, M. and Snyder, M. (1998). Pheromone-regulated genes required for yeast mating differentiation. J. Cell Biol. 140, 461-483. doi:10. 1083/jcb.140.3.461

Fleissner, A. and Herzog, S. (2016). Signal exchange and integration during selffusion in filamentous fungi. Semin. Cell Dev. Biol. 57, 76-83. doi:10.1016/j. semcdb.2016.03.016

Fleissner, A., Diamond, S. and Glass, N. L. (2009a). The Saccharomyces cerevisiae PRM1 homolog in Neurospora crassa is involved in vegetative and sexual cell fusion events but also has postfertilization functions. Genetics 181 497-510. doi:10.1534/genetics.108.096149

Fleissner, A., Leeder, A. C., Roca, M. G., Read, N. D. and Glass, N. L. (2009b) Oscillatory recruitment of signaling proteins to cell tips promotes coordinated behavior during cell fusion. Proc. Natl. Acad. Sci. USA 106, 19387-19392. doi:10. 1073/pnas.0907039106

Fu, C. and Heitman, J. (2017). PRM1 and KAR5 function in cell-cell fusion and karyogamy to drive distinct bisexual and unisexual cycles in the Cryptococcus pathogenic species complex. PLoS Genet. 13, e1007113. doi:10.1371/journal. pgen.1007113

Fujimura, H. A. (1994). Yeast homolog of mammalian mitogen-activated protein kinase, FUS3/DAC2 kinase, is required both for cell fusion and for G1 arrest of the cell cycle and morphological changes by the cdc37 mutation. J. Cell Sci. 107 2617-2622.

Gallo Castro, D. and Martin, S. G. (2018). Differential GAP requirement for Cdc42 GTP polarization during proliferation and sexual reproduction. J. Cell Biol. doi:10 $1101 / 338186$

Gammie, A. E., Brizzio, V. and Rose, M. D. (1998). Distinct morphological phenotypes of cell fusion mutants. Mol. Biol. Cell 9, 1395-1410. doi:10.1091/mbc 9.6.1395

Gelin-Licht, R., Paliwal, S., Conlon, P., Levchenko, A. and Gerst, J. E. (2012) Scp160-dependent mRNA trafficking mediates pheromone gradient sensing and chemotropism in yeast. Cell Rep. 1, 483-494 doi:10.1016/j.celrep.2012.03.004

Goodenough, U. and Heitman, J. (2014). Origins of eukaryotic sexual reproduction. Cold Spring Harb. Perspect. Biol. 6, a016154. doi:10.1101/ cshperspect.a016154

Goryachev, A. B., Lichius, A., Wright, G. D. and Read, N. D. (2012). Excitable behavior can explain the "ping-pong" mode of communication between cells using the same chemoattractant. BioEssays 34, 259-266. doi:10.1002/bies.201100135 Guo, M., Aston, C., Burchett, S. A., Dyke, C., Fields, S., Rajarao, S. J. R., Uetz, P., Wang, Y., Young, K. and Dohlman, H. G. (2003). The yeast G protein alpha 
subunit Gpa1 transmits a signal through an RNA binding effector protein Scp160. Mol. Cell 12, 517-524. doi:10.1016/S1097-2765(03)00307-1

Hall, A. E. and Rose, M. D. (2019). Cell fusion in yeast is negatively regulated by components of the cell wall integrity pathway. Mol. Biol. Cell 30, 441-452. doi:10. 1091/mbc.E18-04-0236

Hegemann, B., Unger, M., Lee, S. S., Stoffel-Studer, I., van den Heuvel, J., Pelet, S., Koeppl, H. and Peter, M. (2015). A cellular system for spatial signal decoding in chemical gradients. Dev. Cell 35, 458-470. doi:10.1016/j.devcel.2015.10.013

Heiman, M. G. and Walter, P. (2000). Prm1p, a pheromone-regulated multispanning membrane protein, facilitates plasma membrane fusion during yeast mating. J. Cell Biol. 151, 719-730. doi:10.1083/jcb.151.3.719

Heiman, M. G., Engel, A. and Walter, P. (2007). The Golgi-resident protease Kex2 acts in conjunction with Prm1 to facilitate cell fusion during yeast mating. J. Cell Biol. 176, 209-222. doi:10.1083/jcb.200609182

Henderson, N. T., Clark-Cotton, M. R., Zyla, T. R. and Lew, D. J. (2018). How yeast cells find their mate. BioRxiv, 422790. doi:10.1101/422790

Hernández, J. M. and Podbilewicz, B. (2017). The hallmarks of cell-cell fusion Development 144, 4481-4495. doi:10.1242/dev.155523

Hirota, K., Tanaka, K., Watanabe, Y. and Yamamoto, M. (2001). Functiona analysis of the $\mathrm{C}$-terminal cytoplasmic region of the $\mathrm{M}$-factor receptor in fission yeast. Genes Cells 6, 201-214. doi:10.1046/j.1365-2443.2001.00415.x

Huberman, L. B. and Murray, A. W. (2014). A model for cell wall dissolution in mating yeast cells: polarized secretion and restricted diffusion of cell wall remodeling enzymes induces local dissolution. PLoS One 9, e109780. doi:10. 1371/journal.pone.0109780

Insall, R. (2013). The interaction between pseudopods and extracellular signalling during chemotaxis and directed migration. Curr. Opin. Cell Biol. 25, 526-531. doi:10.1016/j.ceb.2013.04.009

Ismael, A., Tian, W., Waszczak, N., Wang, X., Cao, Y., Suchkov, D., Bar, E., Metodiev, M. V., Liang, J., Arkowitz, R. A. et al. (2016). Gbeta promotes pheromone receptor polarization and yeast chemotropism by inhibiting receptor phosphorylation. Sci. Signal. 9, ra38. doi:10.1126/scisignal.aad4376

Jackson, C. L., Konopka, J. B. and Hartwell, L. H. (1991). S. cerevisiae alpha pheromone receptors activate a novel signal transduction pathway for mating partner discrimination. Cell 67, 389-402. doi:10.1016/0092-8674(91)90190-A

Jin, T. (2013). Gradient sensing during chemotaxis. Curr. Opin. Cell Biol. 25 532-537. doi:10.1016/j.ceb.2013.06.007

Jin, H., Carlile, C., Nolan, S. and Grote, E. (2004). Prm1 prevents contactdependent lysis of yeast mating pairs. Eukaryot. Cell 3, 1664-1673. doi:10.1128/ EC.3.6.1664-1673.2004

Jin, H., McCaffery, J. M. and Grote, E. (2008). Ergosterol promotes pheromone signaling and plasma membrane fusion in mating yeast. J. Cell Biol. 180, 813-826. doi:10.1083/jcb.200705076

Jonkers, W., Leeder, A. C., Ansong, C., Wang, Y., Yang, F., Starr, T. L., Camp D. G., III, Smith, R. D. and Glass, N. L. (2014). HAM-5 functions as a MAP kinase scaffold during cell fusion in Neurospora crassa. PLoS Genet. 10, e1004783. doi:10.1371/journal.pgen.1004783

Khalili, B., Merlini, L., Vincenzetti, V., Martin, S. G. and Vavylonis, D. (2018) Exploration and stabilization of Ras1 mating zone: a mechanism with positive and negative feedbacks. PLoS Comput. Biol. 14, e1006317. doi:10.1371/journal.pcbi. 1006317

Kim, K.-M., Lee, Y.-H., Akal-Strader, A., Uddin, M. S., Hauser, M., Naider, F. and Becker, J. M. (2012). Multiple regulatory roles of the carboxy terminus of Ste2p a yeast GPCR. Pharmacol. Res. 65, 31-40. doi:10.1016/j.phrs.2011.11.002

Kurahashi, H., Imai, Y. and Yamamoto, M. (2002). Tropomyosin is required for the cell fusion process during conjugation in fission yeast. Genes Cells 7, 375-384 doi:10.1046/j.1365-2443.2002.00526.x

Li, E., Cismowski, M. J. and Stone, D. E. (1998). Phosphorylation of the pheromone-responsive Gbeta protein of Saccharomyces cerevisiae does not affect its mating-specific signaling function. Mol. Gen. Genet. 258, 608-618. doi:10.1007/s004380050774

Lichius, A., Goryachev, A. B., Fricker, M. D., Obara, B., Castro-Longoria, E. and Read, N. D. (2014). CDC-42 and RAC-1 regulate opposite chemotropisms in Neurospora crassa. J. Cell Sci. 127, 1953-1965. doi:10.1242/jcs.141630

Maeder, C. I., Hink, M. A., Kinkhabwala, A., Mayr, R., Bastiaens, P. I. H. and Knop, M. (2007). Spatial regulation of Fus3 MAP kinase activity through a reaction-diffusion mechanism in yeast pheromone signalling. Nat. Cell Biol. 9 , 1319-1326. doi:10.1038/ncb1652

Martin, S. G. (2015). Spontaneous cell polarization: Feedback control of Cdc42 GTPase breaks cellular symmetry. BioEssays 37, 1193-1201. doi:10.1002/bies. 201500077

Martin, S. G. (2016). Role and organization of the actin cytoskeleton during cell-cell fusion. Semin. Cell Dev. Biol. 60, 121-126. doi:10.1016/j.semcdb.2016.07.025

Martin, S. G. and Arkowitz, R. A. (2014). Cell polarization in budding and fission yeasts. FEMS Microbiol. Rev. 38, 228-253. doi:10.1111/1574-6976.12055

McClure, A. W., Minakova, M., Dyer, J. M., Zyla, T. R., Elston, T. C. and Lew, D. J. (2015). Role of polarized $G$ protein signaling in tracking pheromone gradients. Dev. Cell 35, 471-482. doi:10.1016/j.devcel.2015.10.024

Merlini, L., Dudin, O. and Martin, S. G. (2013). Mate and fuse: how yeast cells do it. Open Biol. 3, 130008. doi:10.1098/rsob.130008
Merlini, L., Khalili, B., Bendezú, F. O., Hurwitz, D., Vincenzetti, V., Vavylonis, D. and Martin, S. G. (2016). Local pheromone release from dynamic polarity sites underlies cell-cell pairing during yeast mating. Curr. Biol. 26, 1117-1125. doi:10. 1016/j.cub.2016.02.064

Merlini, L., Khalili, B., Dudin, O., Michon, L., Vincenzetti, V. and Martin, S. G. (2018). Inhibition of Ras activity coordinates cell fusion with cell-cell contact during yeast mating. J. Cell Biol. 217, 1467-1483. doi:10.1083/jcb.201708195

Metodiev, M. V., Matheos, D., Rose, M. D. and Stone, D. E. (2002). Regulation of MAPK function by direct interaction with the mating-specific Galpha in yeast. Science 296, 1483-1486. doi:10.1126/science.1070540

Moore, T. I., Chou, C.-S., Nie, Q., Jeon, N. L. and Yi, T.-M. (2008). Robust spatial sensing of mating pheromone gradients by yeast cells. PLoS ONE 3, e3865. doi:10.1371/journal.pone.0003865

Moore, T. I., Tanaka, H., Kim, H. J., Jeon, N. L. and Yi, T.-M. (2013). Yeast G-proteins mediate directional sensing and polarization behaviors in response to changes in pheromone gradient direction. Mol. Biol. Cell 24, 521-534. doi:10. 1091/mbc.e12-10-0739

Muller, E. M., Mackin, N. A., Erdman, S. E. and Cunningham, K. W. (2003). Fig1p facilitates $\mathrm{Ca} 2+$ influx and cell fusion during mating of Saccharomyces cerevisiae. J. Biol. Chem. 278, 38461-38469. doi:10.1074/jbc.M304089200

Muller, N., Piel, M., Calvez, V., Voituriez, R., Gonçalves-Sá, J., Guo, C.-L., Jiang, X., Murray, A. and Meunier, N. (2016). A predictive model for yeast cell polarization in pheromone gradients. PLoS Comput. Biol. 12, e1004795. doi:10. 1371/journal.pcbi.1004795

Nern, A. and Arkowitz, R. A. (1998). A GTP-exchange factor required for cell orientation. Nature 391, 195-198. doi:10.1038/34458

Nern, A. and Arkowitz, R. A. (1999). A Cdc24p-Far1p-Gbetagamma protein complex required for yeast orientation during mating. J. Cell Biol. 144, 1187-1202. doi:10.1083/jcb.144.6.1187

Nern, A. and Arkowitz, R. A. (2000). G proteins mediate changes in cell shape by stabilizing the axis of polarity. Mol. Cell 5, 853-864. doi:10.1016/S10972765(00)80325-1

Olmo, V. N. and Grote, E. (2010a). Prm1 functions as a disulfide-linked complex in yeast mating. J. Biol. Chem. 285, 2274-2283. doi:10.1074/jbc.M109.068874

Olmo, V. N. and Grote, E. (2010b). Prm1 targeting to contact sites enhances fusion during mating in Saccharomyces cerevisiae. Eukaryot. Cell 9, 1538-1548. doi:10. 1128/EC.00116-10

Palanivelu, R. and Preuss, D. (2000). Pollen tube targeting and axon guidance: parallels in tip growth mechanisms. Trends Cell Biol. 10, 517-524. doi:10.1016/ S0962-8924(00)01849-3

Paliwal, S., Iglesias, P. A., Campbell, K., Hilioti, Z., Groisman, A. and Levchenko, A. (2007). MAPK-mediated bimodal gene expression and adaptive gradient sensing in yeast. Nature 446, 46-51. doi:10.1038/nature05561

Palma-Guerrero, J., Leeder, A. C., Welch, J. and Glass, N. L. (2014). Identification and characterization of LFD1, a novel protein involved in membrane merger during cell fusion in Neurospora crassa. Mol. Microbiol. 92, 164-182. doi:10.1111/mmi.12545

Paterson, J. M., Ydenberg, C. A. and Rose, M. D. (2008). Dynamic localization of yeast Fus $2 p$ to an expanding ring at the cell fusion junction during mating. J. Cell Biol. 181, 697-709. doi:10.1083/jcb.200801101

Petersen, J., Weilguny, D., Egel, R. and Nielsen, O. (1995). Characterization of fus 1 of Schizosaccharomyces pombe: a developmentally controlled function needed for conjugation. Mol. Cell. Biol. 15, 3697-3707. doi:10.1128/MCB.15.7.3697

Petersen, J., Nielsen, O., Egel, R. and Hagan, I. M. (1998). FH3, a domain found in formins, targets the fission yeast formin Fus1 to the projection tip during conjugation. J. Cell Biol. 141, 1217-1228. doi:10.1083/jcb.141.5.1217

Philips, J. and Herskowitz, I. (1997). Osmotic balance regulates cell fusion during mating in Saccharomyces cerevisiae. J. Cell Biol. 138, 961-974. doi:10.1083/jcb. 138.5.961

Pryciak, P. M. and Huntress, F. A. (1998). Membrane recruitment of the kinase cascade scaffold protein Ste5 by the Gbetagamma complex underlies activation of the yeast pheromone response pathway. Genes Dev. 12, 2684-2697. doi:10. 1101/gad.12.17.2684

Rajavel, M., Philip, B., Buehrer, B. M., Errede, B. and Levin, D. E. (1999). Mid2 is a putative sensor for cell integrity signaling in Saccharomyces cerevisiae. Mol. Cell. Biol. 19, 3969-3976. doi:10.1128/MCB.19.6.3969

Roca, M. G., Arlt, J., Jeffree, C. E. and Read, N. D. (2005). Cell biology of conidial anastomosis tubes in Neurospora crassa. Eukaryot. Cell 4, 911-919. doi:10.1128/ EC.4.5.911-919.2005

Segall, J. E. (1993). Polarization of yeast cells in spatial gradients of alpha mating factor. Proc. Natl. Acad. Sci. USA 90, 8332-8336. doi:10.1073/pnas.90.18.8332 Serrano, A., Illgen, J., Brandt, U., Thieme, N., Letz, A., Lichius, A., Read, N. D. and Fleissner, A. (2018). Spatio-temporal MAPK dynamics mediate cell behavio coordination during fungal somatic cell fusion. J. Cell Sci. 131, jcs213462. doi:10. 1242/jcs.213462

Sharifmoghadam, M. R., de Leon, N., Hoya, M., Curto, M.-A. and Valdivieso, M.-H. (2010). Different steps of sexual development are differentially regulated by the Sec8p and Exo70p exocyst subunits. FEMS Microbiol. Lett. 305, 71-80 doi:10.1111/j.1574-6968.2010.01915.x 
Sheltzer, J. M. and Rose, M. D. (2009). The class V myosin Myo2p is required for Fus $2 \mathrm{p}$ transport and actin polarization during the yeast mating response. Mol. Biol. Cell 20, 2909-2919. doi:10.1091/mbc.e08-09-0923

Shi, C., Kaminskyj, S., Caldwell, S. and Loewen, M. C. (2007). A role for a complex between activated $\mathrm{G}$ protein-coupled receptors in yeast cellular mating. Proc. Natl. Acad. Sci. USA 104, 5395-5400. doi:10.1073/pnas.0608219104

Smith, J. A., Hall, A. E. and Rose, M. D. (2017). Membrane curvature directs the localization of Cdc42p to novel foci required for cell-cell fusion. J. Cell Biol. 216, 3971-3980. doi:10.1083/jcb.201703169

Sourjik, V. and Wingreen, N. S. (2012). Responding to chemical gradients: bacterial chemotaxis. Curr. Opin. Cell Biol. 24, 262-268. doi:10.1016/j.ceb.2011. 11.008

Stein, R. A., Smith, J. A. and Rose, M. D. (2015). An amphiphysin-like domain in Fus2p is required for Rvs161p interaction and cortical localization. G3 (Bethesda) 6, 337-349. doi:10.1534/g3.115.023960

Strickfaden, S. C. and Pryciak, P. M. (2008). Distinct roles for two Galpha-Gbeta interfaces in cell polarity control by a yeast heterotrimeric $\mathrm{G}$ protein. Mol. Biol. Cell 19, 181-197. doi:10.1091/mbc.e07-04-0385

Suchkov, D. V., DeFlorio, R., Draper, E., Ismael, A., Sukumar, M., Arkowitz, R. and Stone, D. E. (2010). Polarization of the yeast pheromone receptor requires its internalization but not actin-dependent secretion. Mol. Biol. Cell 21, 1737-1752. doi:10.1091/mbc.e09-08-0706

van Drogen, F., Stucke, V. M., Jorritsma, G. and Peter, M. (2001). MAP kinase dynamics in response to pheromones in budding yeast. Nat. Cell Biol. $\mathbf{3}$, 1051-1059. doi:10.1038/ncb1201-1051

Vjestica, A., Merlini, L., Dudin, O., Bendezu, F. O. and Martin, S. G. (2016). Microscopy of fission yeast sexual lifecycle. J. Vis. Exp., 109, e53801. doi:10. $3791 / 53801$
Vjestica, A., Merlini, L., Nkosi, P. J. and Martin, S. G. (2018). Gamete fusion triggers bipartite transcription factor assembly to block re-fertilization. Nature $\mathbf{5 6 0}$, 397-400. doi:10.1038/s41586-018-0407-5

Wang, Y., Marotti, L. A., Jr., Lee, M. J. and Dohlman, H. G. (2005). Differential regulation of $\mathrm{G}$ protein alpha subunit trafficking by mono- and polyubiquitination. J. Biol. Chem. 280, 284-291. doi:10.1074/jbc.M411624200

Weichert, M., Lichius, A., Priegnitz, B.-E., Brandt, U., Gottschalk, J., Nawrath, T., Groenhagen, U., Read, N. D., Schulz, S. and Fleissner, A. (2016). Accumulation of specific sterol precursors targets a MAP kinase cascade mediating cell-cell recognition and fusion. Proc. Natl. Acad. Sci. USA 113, 11877-11882. doi:10.1073/ pnas. 1610527113

Yamamoto, T., Mochida, J., Kadota, J., Takeda, M., Bi, E. and Tanaka, K. (2010). Initial polarized bud growth by endocytic recycling in the absence of actin cabledependent vesicle transport in yeast. Mol. Biol. Cell 21, 1237-1252. doi: 10.1091/ mbc.E09-05-0412

Ydenberg, C. A., Stein, R. A. and Rose, M. D. (2012). Cdc42p and Fus2p act together late in yeast cell fusion. Mol. Biol. Cell 23, 1208-1218. doi:10.1091/mbc. e11-08-0723

Zhang, N.-N., Dudgeon, D. D., Paliwal, S., Levchenko, A., Grote, E. and Cunningham, K. W. (2006). Multiple signaling pathways regulate yeast cell death during the response to mating pheromones. Mol. Biol. Cell 17, 3409-3422. doi:10. 1091/mbc.e06-03-0177

Zhu, M., Torres, M. P., Kelley, J. B., Dohlman, H. G. and Wang, Y. (2011). Pheromone- and RSP5-dependent ubiquitination of the $\mathrm{G}$ protein beta subunit Ste4 in yeast. J. Biol. Chem. 286, 27147-27155. doi:10.1074/jbc.M111.254193 